\title{
SYMPOSIUM
}

\section{Frontal contributions to face processing differences in autism: Evidence from fMRI of inverted face processing}

\author{
SUSAN Y. BOOKHEIMER,${ }^{1,2}$ A. TING WANG,${ }^{3}$ ASHLEY SCOTT, ${ }^{1}$ MARIAN SIGMAN, ${ }^{1,2}$ \\ AND MIRELLA DAPRETTO ${ }^{1}$ \\ ${ }^{1}$ Department of Psychiatry and Biobehavioral Sciences, David Geffen School of Medicine, University of California Los Angeles, \\ Los Angeles, California \\ ${ }^{2}$ Department of Psychology, University of California Los Angeles, Los Angeles, California \\ ${ }^{3}$ Department of Psychiatry, Mount Sinai School of Medicine, New York, New York
}

(Received January 8, 2008; Final Revision August 9, 2008; AccePted August 11, 2008)

\begin{abstract}
Functional neuroimaging studies of face processing deficits in autism have typically focused on visual processing regions, such as the fusiform face area (FFA), which have shown reduced activity in autism spectrum disorders (ASD), though inconsistently. We recently reported reduced activity in the inferior frontal region in ASD, implicating impaired mirror-neuron systems during face processing. In the present study, we used fMRI during a face processing task in which subjects had to match faces presented in the upright versus inverted position. Typically developing (TD) children showed a classic behavioral inversion effect, increased reaction time for inverted faces, while this effect was significantly reduced in ASD subjects. The fMRI data showed similar responses in the fusiform face area for ASD and TD children, with both groups demonstrating increased activation for inverted faces. However, the groups did differ in several brain regions implicated in social cognition, particularly prefrontal cortex and amygdala. These data suggest that the behavioral differences in processing upright versus inverted faces for TD children are related not to visual information processing but to the social significance of the stimuli. Our results are consistent with other recent studies implicating frontal and limbic dysfunction during face processing in autism. (JINS, 2008, 14, 922-932.)
\end{abstract}

Keywords: Functional MRI, Autism, Asperger's, Face processing, Face inversion, Development

\section{INTRODUCTION}

One of the most consistent findings in children with autism is their reduced interest in human faces. Faces convey critical social information, including the intent of the speaker, eye-gaze cues for directing attention, and information signaling the speaker's emotional state (e.g., Chawarska et al., 2003). Thus, a failure to display normal face interest or face processing may have broad and far-reaching effects on social development in children with autism spectrum disorders (ASD). For these reasons, much recent research into the neurobiology of autism has focused on the extent to which

Correspondence and reprint requests to: Susan Y. Bookheimer, Department of Psychiatry and Biobehavioral Sciences, University of California, Los Angeles, 760 Westwood Plaza, Los Angeles, CA 90095. E-mail: sbook@ucla.edu children with ASD perceive faces normally, that is, as unique entities distinct from other visually recognizable objects.

Numerous studies of children with autism indicate a range of deficits in face processing including impaired face recognition, reduced direct gaze, failure to benefit from gaze cues, impaired perception of emotional expressions, and impaired production of facial expressions for social communication (e.g., Campbell et al., 2006; Dawson et al., 2005). A critical question in understanding face processing deficits in autism is whether they reflect a fundamental difference in the brain's ability to process the visual information necessary for perceiving faces and facial affect, or whether impaired face processing is secondary to another deficit, such as reduced social interest, increased emotional discomfort with emotional expressions, general attention deficits, or strategic differences in how attention is directed toward face stimuli. 
One source of evidence citing the uniqueness of face versus object processing come from studies on the "face inversion effect," that is, the superiority in recognizing or matching faces presented in an upright versus an inverted orientation. The inversion effect is much greater for faces than for other objects like houses, presumably because one cannot easily extract a face "schema" from an inverted presentation (Farah et al., 1991, 1995a); we expect faces to be in a typical orientation and have specialized mechanisms for processing them. Inverted faces provide an interesting contrast for upright face processing studies because the information is visually identical, unlike comparisons with, for example, objects, which have many featural differences (Farah et al., 1995a). Inversion is thought to reduce holistic processing that is essential for face identity (Farah et al., 1991; Yin, 1969). Several behavioral studies of inverted face processing in individuals with autism indicate that they may have a smaller "inversion effect," that is, they show a proportionally smaller decrement in performance when faces are inverted as compared to when objects are inverted (Ashwin et al., 2006; Barton et al., 2001; Hobson et al., 1988). This pattern may reflect a deficit in the visual processing strategy of seeing faces as a learned schema, relying instead on serial or piecemeal analysis of individual details of a face (Behrmann et al., 2006). While such a strategy is inefficient in processing upright faces, it is less so in processing inverted faces, thus potentially explaining the reduced inversion effect in autism. For instance, Barton et al. (2007) varied the spatial orientation of eyes and mouths with internal features like eye and mouth color using an inversion paradigm; inversion affected primarily the spatial changes, which were much harder to detect than the internal detail changes, supporting the notion that inversion decreases the ability to process faces configurally, without affecting detail processing. Furthermore, they found that ASD children with more severe face processing deficits showed a significantly smaller inversion effect than ASD children without these deficits, who in turn had a smaller inversion effect than controls. Such findings are consistent with a wealth of data implicating a preference for local processing in children with autism (Happe \& Frith, 2006; Mottron et al., 1999). In contrast, Rose et al. (2007) found that, unlike control subjects, ASD children did not show a performance decrement to neutral faces that were inverted compared with upright, although they showed a large performance decline when upright faces contained emotional expressions. It is unknown whether differences in processing upright versus inverted faces in autism reflect different visual processing mechanisms, likely represented in lower level or visual association cortices (Pellicano et al., 2007), or whether they represent differences in strategies engaged during face processing, which might be expected to engage more frontal lobe mechanisms.

Several prior functional imaging studies have examined the effect of inversion in visual processing of faces versus other objects in normal individuals. Using fMRI, Haxby et al. (1999) examined responses in face selective and house selective visual brain regions; inversion produced increased activation in the house areas for inverted faces, but inverted houses produced no additional activity in the "fusiform face area" (FFA), that part of inferior temporal-occipital cortex specialized for face processing. A similar finding was reported by Aguirre et al. (1999). Yovel and Kanwisher (2004) varied whether subjects had to attend to configuration or parts information in faces presented in either the upright or inverted orientation. They found less activation in the right FFA for inverted faces regardless of processing mode. They surmised that, for adults, the right FFA responds to face content and is not dependent upon any potential strategies in configural versus detail processing. In autism, neuroimaging studies have yielded mixed results on defining face processing impairment. Initial reports suggested that brain activation in the FFA was reduced in ASD compared with controls (Pierce et al., 2001; Schultz et al., 2000). For instance, Schultz et al. (2000) found face-related fMRI activation in autism was in the part of the fusiform gyrus associated with object processing, while controls clearly showed a differentiation between more medial fusiform object processing areas and the more lateral face areas. Pierce et al. (2001) also showed reduced FFA area activity in autism compared with controls, suggesting a breakdown of this system or a failure to develop face expertise while Hadjikhani et al. (2004) found no group differences in the FFA. A study by Pierce et al. (2004) demonstrated that ASD children could evidence ample face area activity when presented with familiar faces like those of family members, suggesting that the face area was intact, but that ASD children were uninterested in or avoidant of faces they did not recognize. In a carefully conducted experiment in which face scanning and gaze fixation was measured, Dalton et al. (2005) demonstrated that differences in fusiform gyrus activity among children with autism were best attributed to differences in how the faces were scanned, and not to differences in which brain regions were used for face processing. Similarly, Piggot et al. (2004), Wang et al. (2004), and others found that when task demands required subjects to maintain their gaze on the faces, ASD children showed activity similar to that of controls in face regions. In addition, differences between ASD and control subjects in tasks involving observation and imitation of facial affect have been found in frontal and limbic regions associated with the mirror neuron system (Dapretto et al., 2006), suggesting that face processing deficits in autism may have less to do with visual processing, but may rather be explained by differences in brain regions important in processing the unique significance of faces such as the amygdala, inferior frontal and medial prefrontal cortex, known collectivity as "social brain" regions. No studies to date have examined the neural basis of how children with autism process inverted faces in comparison to typically oriented faces. In this study we used fMRI while ASD and TD children matched a probe face to one of two similar target faces in conditions where the probe was presented in either the upright or inverted position. We sought to determine whether there was a behav- 
Table 1. Participant characteristics

\begin{tabular}{|c|c|c|c|c|c|c|c|c|c|}
\hline & \multicolumn{3}{|c|}{ Chronological age } & \multicolumn{3}{|c|}{$\begin{array}{c}\text { PPVT } \\
\text { (age equivalent scores }\end{array}$} & \multicolumn{3}{|c|}{$\begin{array}{l}\text { WISCIII vocabulary } \\
\text { (age scaled scores) }\end{array}$} \\
\hline & Min & Max & Mean $(S D)$ & Min & Max & Mean $(S D)$ & Min & Max & Mean $(S D)$ \\
\hline ASD & 7.8 & 19.6 & $11.3(4.0)$ & 6.9 & 18.6 & $11.7(4.7)$ & 6.3 & 13.8 & $9.8(2.5)$ \\
\hline TD & 8.1 & 15.7 & $11.9(2.4)$ & 9.1 & 22.0 & $16.7(5.6)$ & 9.5 & 16.8 & $13.6(2.7)$ \\
\hline
\end{tabular}

ioral difference in the inversion effect and whether group differences in brain activation during the inversion task were predominant in visual association regions (medial and lateral fusiform) or in frontal brain regions. Specifically, we were interested in examining which brain regions would differentiate ASD from TD children during upright and inverted face processing.

\section{METHODS}

\section{Participants}

Twelve males with autism spectrum disorder (ASD) and twelve age-matched typically developing (TD) males participated in the study. Children and adolescents with ASD were recruited from the UCLA autism clinic, regional centers, and parent advocacy groups in the greater Los Angeles area, while TD children were recruited from the UCLA community and local public schools. Individuals with ASD had received a previous clinical diagnosis of ASD (autism, Asperger's, or PDD-NOS), which we confirmed using both the Autism Diagnostic Interview-Revised (ADI-R) (Lord et al., 1994) and the Autism Diagnostic Observation Schedule (ADOS) (Lord et al., 2000). Of the 12 children with ASD, two were diagnosed with PDD-NOS, two with Asperger's, and eight with autism. The ADOS combined social and communication scores ranged from 7 to 19 (average $=$ 11.3), and of the 12 ASD subjects, five met ADOS criteria for autism spectrum disorder while seven met the narrower criteria for autism. On the ADI-R, all met criteria for autism except one Asperger's subject whose symptoms appeared after 36 months. Exclusionary criteria included the presence of any known neurological or genetic disorders (e.g., epilepsy, Fragile X), clear structural brain abnormalities (e.g., aneurysm) or a major psychiatric disorder other than autism. In addition, we used the Social Communication Questionnaire (SCQ; Berument et al., 1999) to screen for autistic symptomatology in TD children and adolescents. Descriptive information for the sample, including chronological age, receptive and expressive language age assessed using the Peabody Picture Vocabulary Test-Third Edition (PPVT-III; Dunn \& Dunn, 1997) and the vocabulary subtest of the Wechsler Intelligence Scale for Children (Wechsler, 1991), respectively, is shown in Table 1. The groups did not differ significantly in chronological age, although there was a greater variation in age among the ASD group, due largely to a single outlier aged 19. TD children had higher receptive and expressive language ages than children with ASD, as expected in this disorder. All subjects and parents provided informed consent to participate; the study was approved by the UCLA human subjects Internal Review Board.

\section{Experimental Task}

Participants performed three matching tasks while undergoing fMRI. Examples of stimuli are shown in Figure 1. In the two experimental conditions, subjects viewed neutral faces from a standard series (Ekman \& Friesen, 1976) and selected one of two choices to match a target face presented at the top of the screen. In the upright face condition, all faces were presented upright, whereas in the inverted con-

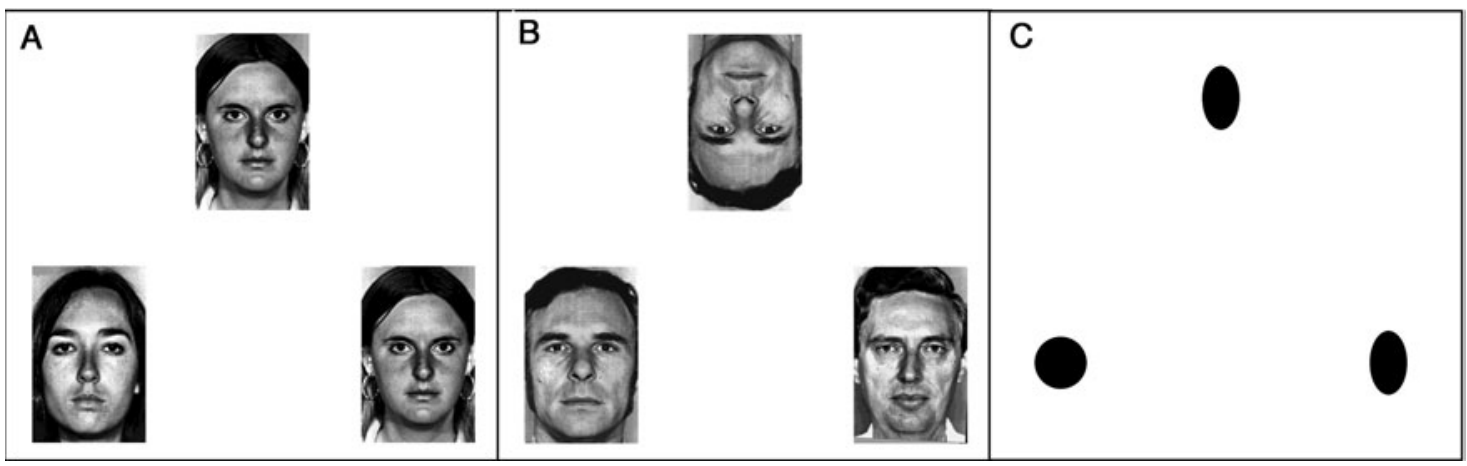

Fig. 1. Examples of stimuli used in the upright condition (A), the inverted condition (B), and the forms condition (C). Participants were asked to choose one of the two bottom faces or forms to match the target at the top. 
dition, the target face at the top of screen was inverted. In the control "forms" condition, participants chose one of two oval shapes to match the target form at the top. This condition controlled for attentional and response aspects of the task but provided only very simple visual information to avoid masking any potential visual processing differences between groups. Subjects indicated their judgments by pressing a button on a magnet compatible response pad.

The paradigm consisted of nine activation blocks: two blocks each of upright faces and inverted faces alternated with five forms control blocks. The order of presentation of conditions was counterbalanced across subjects within each group. Each block consisted of six trials, which were $5 \mathrm{~s}$ each, presented in random order. The functional scan lasted $4 \mathrm{~min}$ and $53 \mathrm{~s}$.

\section{Data Acquisition}

Images were acquired with a GE 3-Tesla scanner with an Advanced NMR upgrade (Wilmington, MA) for echoplanar imaging. T2-weighted sagittal scout images were used to prescribe the planes of the functional scans in each subject. High-resolution structural images covering the entire cerebral volume were acquired to allow for spatial registration of the functional images, using an echo-planar, spinecho sequence $(\mathrm{TR}=4000 \mathrm{~ms} ; \mathrm{TE}=54 \mathrm{~ms}$; flip angle $=$ $90^{\circ}$; matrix size $\left.=128 \times 128 ; \mathrm{FOV}=20 \mathrm{~cm} ; 4 \mathrm{NEX}\right)$. The functional scan was composed of 117 volumes acquired over 16 axial slices ( $4 \mathrm{~mm}$ thick/1 mm gap) using an asymmetric spin-echo sequence to reduce susceptibility artifact in the area of the amygdala $(\mathrm{TR}=2500 \mathrm{~ms}$; $\mathrm{TE}=70 \mathrm{~ms}$; offset $=25 \mathrm{~ms}$; flip angle $=180^{\circ}$; matrix size $=64 \times 64$; $\mathrm{FOV}=20 \mathrm{~cm} ; 1 \mathrm{NEX}$ ).

To correct for head motion, functional images were realigned using Automated Image Registration (Woods et al., 1998). Images were then spatially normalized into a standard reference space (Talairach \& Tournoux, 1988) using polynomial nonlinear warping to allow for intersubject averaging. Finally, all images were smoothed using a 6-mm Gaussian kernel (full-width/half-maximum) to increase signal-to-noise ratio.

\section{Data Analysis}

Data were analyzed using a two step approach in which whole-brain data were first analyzed within group, and between groups effects were assessed using a region of interest approach to reduce errors due to multiple comparisons.

For within-group analysis, imaging data were analyzed using statistical parametric mapping (SPM99; Wellcome Department of Cognitive Neurology, London, UK) according to the general linear model with a box-car reference function to compensate for the lag in hemodynamic response. Activation maps for comparisons of interest (upright v. forms, inverted v. forms) were generated for each participant. The resulting contrast images were then entered into randomeffects analyses. For each group, a one-sample $t$ test was used to examine activation in each activation versus forms comparison. Thresholds of significance were set at $p<.05$ (Bonferroni corrected for multiple comparisons within SPM) for magnitude and $p<.05$ (corrected for spatial extent using Gaussian random fields estimation and corresponding to a cluster size of 20 voxels).

Between-group differences were then examined in regions of interest (ROIs) selected based on a priori hypotheses (fusiform gyrus and prefrontal cortex). ROIs were defined functionally as the voxels statistically significantly activated in either group in the all face activation versus forms comparison. These voxels were chosen by performing a global analysis for both TD and ASD groups, using all conditions (upright and inverted) compared with the forms control task, and extracting all voxels identified in a contiguous region in the left and right fusiform and prefrontal cortices. This approach allows for a functional ROI selection that is unbiased with respect to group membership and to experimental condition. The precise localization of the regions, therefore, were defined by the data themselves and not by an a priori assumption of the precise location of the region, and represented in each case the largest cluster of voxels in the fusiform gyrus and the inferior frontal gyrus. The fusiform gyrus ROIs' were centered around Talairach coordinates $-38,-56,-10(\mathrm{LH})$ and $30,-44,-12(\mathrm{RH})$. Frontal ROIS extended from $-46,10,32(\mathrm{LH})$ and 34, 10, $32(\mathrm{RH})$. We also performed a post hoc identification of an ROI in the precuneus $(28,-66,36)$, as it was the sole region showing a strong activation exclusively in the ASD group. This region was identified on the basis of the contrast between all faces and the forms task only in the ASD sample. The regions of interest defined from the group activation maps were then applied to each subject's raw, spatially normalized data. Images corrupted by spikes or by large intensity variations due to motion were removed before analysis using a conservative algorithm that eliminated extreme outliers. There were no group differences in the frequency of intensity outliers $(\mathrm{t}=0.23 ; p>.8)$, indicating the subject groups did not differ in image variability due to head motion or spikes. In each ROI, magnitude of activation scores were computed for each subject by averaging the percent signal change in each face condition relative to the forms condition using the raw signal intensity values. These scores were then entered as dependent variables in a 2 (group) $\times 2$ (condition) $\times 2$ (region) $\times 2$ (hemisphere) multivariate analysis of variance (ANOVA).

\section{RESULTS}

\section{Behavioral Results}

We acquired accuracy data and response times (RT) during scanning from all 12 children with ASD and 9 TD children (equipment failure accounted for lost data in 3 subjects). With respect to accuracy, there were marked differences in variability between the two subject groups as seen in Table 2 . The Levine's test for equality of variance found significant 
Table 2. Task performance

\begin{tabular}{|c|c|c|c|c|c|c|c|c|}
\hline & \multicolumn{4}{|c|}{ Accuracy (\% correct) } & \multicolumn{4}{|c|}{ Response time (s) } \\
\hline & TD & ASD & $t_{1,19}$ & $p$ & TD & ASD & $t_{1,19}$ & $p$ \\
\hline Upright & $100(0)$ & $93.2(8.1)$ & 2.5 & .02 & $1.62(.30)$ & $1.90(.39)$ & 1.9 & .07 \\
\hline Inverted & $89.7(8.5)$ & $80.3(18.4)$ & 1.4 & .18 & $2.56(.18)$ & $2.54(.36)$ & .84 & .41 \\
\hline Forms & $98.1(3.4)$ & $96.0(5.6)$ & 1.0 & .31 & $1.13(.18)$ & $1.35(.28)$ & 1.8 & .09 \\
\hline
\end{tabular}

Note. Values are presented as mean $(S D)$.

inhomogeneity on variance for both upright $(F=32.4$; $p=$ $.000)$ and inverted ( $F=4.82 ; p=.041)$ accuracy. Inspection of the data showed that, for upright faces, the control group had a strong ceiling effect, with all subjects performing at ceiling (no errors for any subject). For inverted faces, the inhomogeneity of variance was apparently due to a bimodal distribution among the ASD subjects, with three subjects performing at chance $(\mathrm{x}=51 \%)$ and the remaining performing as well as controls (89.7\% controls, $89.9 \%$ ASD). The pronounced ceiling effect for controls made it impossible to determine, based on accuracy, whether there were inversion effect differences between groups. For this reason further analyses focused on the reaction time inversion effect, which passed the Levene's test for equality of variance (all $p$ 's $>.44$ ).

For reaction time data, we included only correct responses in our calculation. A repeated-measures ANOVA yielded a significant effect of Condition $(F(2,38)=212.29 ; p<.001)$, such that Inverted (mean RT $=2.55$ ) was significantly longer than Upright (mean RT $=1.79)$ or Forms (mean RT = 1.26), but no main effects of group and no group $\times$ condition interaction $(F(2,38)=2.178 ; p=.142)$. Planned comparisons revealed that TD children were slightly but not significantly ( $t=2.16 ; p=.44$ uncorrected) faster at matching upright faces and forms control than children with ASD, although the two groups did not differ in response times for the inverted condition $(p>.4)$.

We calculated the reaction time inversion effect as the difference in RT in response to inverted compared with upright faces for each subject. Comparisons between groups revealed that TD children showed a greater inversion effect than did ASD children (944 ms TD; $624 \mathrm{~ms} \mathrm{ASD;} \mathrm{t}=2.45$; $p=0.02$ ). One ASD who performed at chance and responded to only $25 \%$ of the stimuli also had an outlying inversion effect (substantially better performance for inverted faces); however, without this subject the group differences remained significant $(\mathrm{t}=2.28 ; p=.034)$.

There were no significant correlations between age, reaction times, and inversion effect for either group, and no correlations between ADOS scores and any of these measures for ASD subjects (all $p>.05$ ).

\section{fMRI Results}

\section{Within-group SPM results}

Within-group analyses revealed notable similarities in activation patterns during upright and inverted face processing between groups in visual processing regions. Peaks of activation exceeding a magnitude threshold of $p<.05$ (corrected) and a spatial extent threshold of $p<.05$ (correctedcorresponding to a cluster size of 20 voxels) are shown in Table 3. In both the upright versus forms and the inverted versus forms comparisons, children with ASD and TD children showed strong activity in the fusiform gyrus bilaterally. In addition, both TD and ASD children showed strong activation in the Lingual gyrus of both hemispheres. Figure 2 shows areas of visual activity for upright oriented
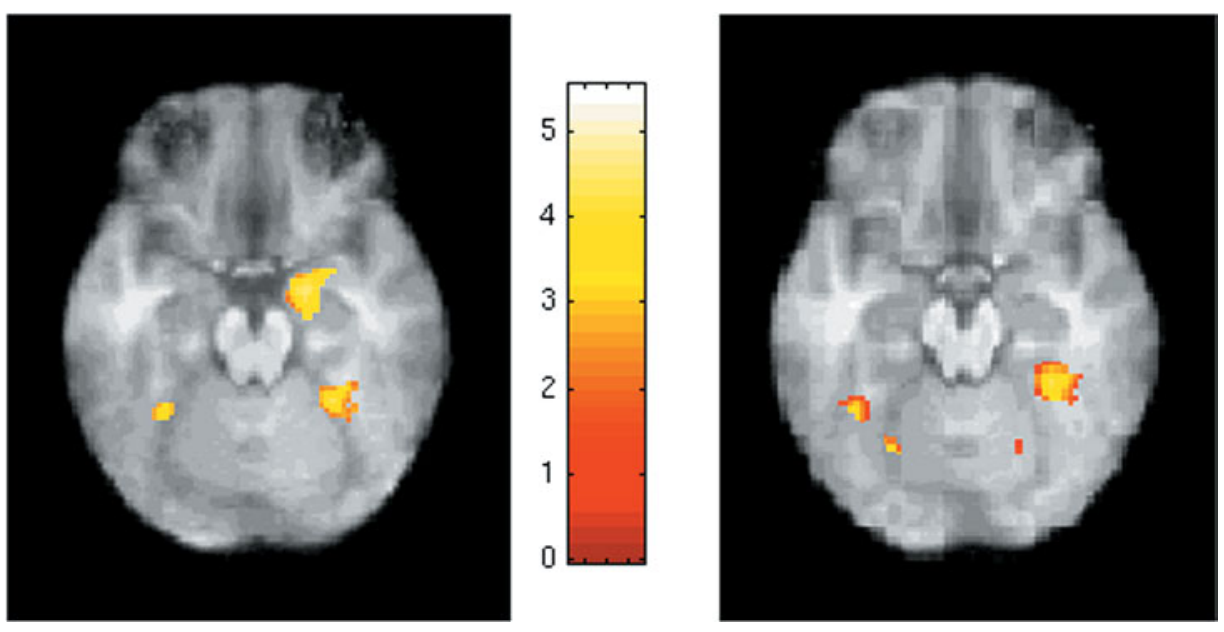

Fig. 2. Statistical Parametric Mapping (SPM) in the ventral cortex during upright face processing ( $v s$. forms matching control). Left, control subjects, right, ASD. Both groups of subjects show bilateral activation in Fusiform gyrus, while only controls show activity in the amygdala. Left side of the image $=$ left hemisphere. Foci of activations are found in Table 3 . 
Table 3. Peaks of activation in whole brain within-groups SPM analysis

\begin{tabular}{|c|c|c|c|c|c|c|c|c|c|c|c|c|c|c|c|c|c|c|}
\hline \multirow[b]{3}{*}{ Region } & \multirow[b]{3}{*}{ BA } & & \multicolumn{8}{|c|}{ Upright $v s$. Forms } & \multicolumn{8}{|c|}{ Inverted $v s$. Forms } \\
\hline & & & \multicolumn{4}{|c|}{ TD } & \multicolumn{4}{|c|}{ ASD } & \multicolumn{4}{|c|}{ TD } & \multicolumn{4}{|c|}{ ASD } \\
\hline & & & $\mathrm{x}$ & $\mathrm{y}$ & $\mathrm{z}$ & $\mathrm{t}$ & $\mathrm{x}$ & $\mathrm{y}$ & $\mathrm{z}$ & $\mathrm{t}$ & $\mathrm{x}$ & $\mathrm{y}$ & $\mathrm{z}$ & $\mathrm{t}$ & $\mathrm{x}$ & $\mathrm{y}$ & $\mathrm{z}$ & $\mathrm{t}$ \\
\hline \multirow[t]{3}{*}{ Lateral fusiform } & 37 & $\mathrm{~L}$ & -40 & -52 & -8 & 6.21 & -34 & -58 & -10 & 7.25 & -36 & -50 & -14 & 8.73 & -38 & -60 & -8 & 9.19 \\
\hline & & $\mathrm{L}$ & & & & & -42 & -48 & -18 & 6.86 & -40 & -60 & -10 & 5.30 & & & & \\
\hline & & $\mathrm{R}$ & 38 & -56 & -10 & 5.47 & 30 & -44 & -12 & 8.03 & 38 & -56 & -10 & 6.82 & 38 & -46 & -8 & 3.88 \\
\hline Medial fusiform & 37 & $\mathrm{R}$ & 22 & -50 & -8 & 7.84 & 24 & -52 & -4 & 4.98 & 30 & -54 & -14 & 8.28 & 32 & -42 & -14 & 9.54 \\
\hline \multirow{2}{*}{ Lingual } & 19 & $\mathrm{~L}$ & -12 & -72 & -4 & 5.57 & -24 & -70 & -8 & 5.08 & -24 & -74 & -6 & 8.21 & -32 & -82 & 24 & 4.06 \\
\hline & & $\mathrm{R}$ & 26 & -64 & 8 & 4.67 & 20 & -68 & -4 & 5.52 & 28 & -68 & -8 & 8.03 & 20 & -68 & -4 & 6.48 \\
\hline \multirow[t]{2}{*}{ IFG } & 44 & $\mathrm{~L}$ & & & & & & & & & -46 & 10 & 32 & 5.37 & & & & \\
\hline & & $\mathrm{R}$ & 38 & 0 & 28 & 5.52 & & & & & 34 & 10 & 32 & 5.54 & & & & \\
\hline \multirow[t]{2}{*}{ MFG } & 46 & $\mathrm{R}$ & 40 & 26 & 20 & 6.07 & & & & & 46 & 28 & 24 & 7.03 & 32 & 18 & 10 & 4.95 \\
\hline & 10 & $\mathrm{R}$ & 32 & 46 & 8 & 5.41 & & & & & & & & & 30 & 52 & 6 & 4.39 \\
\hline \multirow[t]{2}{*}{ Amygdala } & & $\mathrm{R}$ & 20 & 2 & -10 & 5.64 & & & & & & & & & & & & \\
\hline & & $\mathrm{L}$ & -22 & -10 & -10 & 5.18 & & & & & & & & & & & & \\
\hline Precuneus & 7 & $\mathrm{~L}$ & & & & & & & & & & & & & -28 & -66 & 36 & 4.73 \\
\hline
\end{tabular}

Note. Results are corrected for multiple comparisons at both the cluster level (23 voxels, $p<.05)$ and at the magnitude (voxel) level ( $p<.05)$. BA refers to Brodmann's area. $\mathrm{L}$ and $\mathrm{R}$ refer to left and right cerebral hemispheres. $\mathrm{x}, \mathrm{y}$, and $\mathrm{z}$ refer to the Talairach coordinates corresponding to the right-left, anterior-posterior, and superior-inferior dimensions, respectively. The $t$ refers to the highest $t$ score within a cluster. IFG $=$ inferior frontal gyrus; MFG $=$ middle frontal gyrus.

faces in both groups. Separating out maxima in the medial versus lateral fusiform showed similar results between groups, with no apparent differences in either the location or the magnitude of activation between ASD and controls (see Table 3). For both groups of children the magnitude of activation in fusiform cortex was greater in the inverted task compared with the upright task.

In frontal cortex, whole-brain analysis revealed significant activation for TD children in both the upright and inverted tasks. The frontal lobe activations were located in the right posterior inferior frontal gyrus extending into the middle frontal gyrus (Brodmann's areas 44 through 46/9) and in the left hemisphere, in the inferior frontal gyrus (BA 44) (Fig. 3). In children with autism, no significant activation was found using corrected thresholds, though there was subthreshold activation in the right dorsolateral prefrontal cortex (Table 3).

The within-group analyses also revealed two regions that appeared to be specific for the groups. TD children showed activation in the right amygdala for upright faces only, while ASD children activated a region in the precuneus (Table 3 ). Groups also differed in activation in the amygdala; the control children showed bilateral activation in the amygdala for the upright task only, while ASD children showed no activation here, even at reduced thresholds. In one region, the precuneus, children with ASD exclusively activated the left and right precuneus in the inversion relative to the forms task in the whole-brain analysis $(\mathrm{t}=4.06)$.

\section{Between-groups ROI analysis}

A fully factorial model tested the effects of group, region, orientation and hemisphere in the fusiform and PFC regions (Figs. 4, 5). Analysis of variance revealed main effects for Orientation $(F(1,154)=38.73, p=.000)$ and was due to an overall increase in activation for inverted compared with upright faces for all subjects in all regions. We found no significant main effect for Group $(F(1,22)=1.93 ; p=$ .179) but found a significant Group $\times$ Region interaction $(F(1,154)=6.83 ; p=.009)$, and a Group $\times$ Hemisphere interaction $(F(1,154)=5.39 ; p=.021)$; a simple effects test showed this was due to relatively less activation in the left PFC for the ASD group relative to controls $(\mathrm{t}=2.5$; $p<.01$; one-tailed) (see Fig. 5). This difference was found in both upright and inverted conditions; indeed in the upright task, children with autism actually showed a slight decrease in activation in the left PFC (Fig. 5). There were no significant three- or four-way interactions.

The precuneus was the only region showing activation exclusively for the ASD group (Fig. 3); to further examine activity in this region we extracted an ROI based on activation for the ASD group only, across both upright and inverted tasks, and applied this to both groups. The children with ASD activated the left and right precuneus in the inversion relative to the forms task in the whole-brain analysis $(\mathrm{t}=$ 4.06); ROI analysis confirmed a significant difference between ASD and control subjects in the inverted condition $(p<.009)$. However it should be noted that this region was selected post hoc and optimized for the ASD group only, which biases the results in favor of a group difference.

Because three ASD subjects performed at chance behaviorally, we evaluated whether the group differences found in the left PFC would remain without these subjects in the analysis. The between-groups differences remained significant when these outlier subjects were excluded $(\mathrm{t}=3.31$; $p<.004$; one-tailed).

To determine whether the differences we observed in the LPFC could be attributed to other factors, we correlated activation here with age, behavioral performance, and in children with autism, severity based on the ADOS. We also 

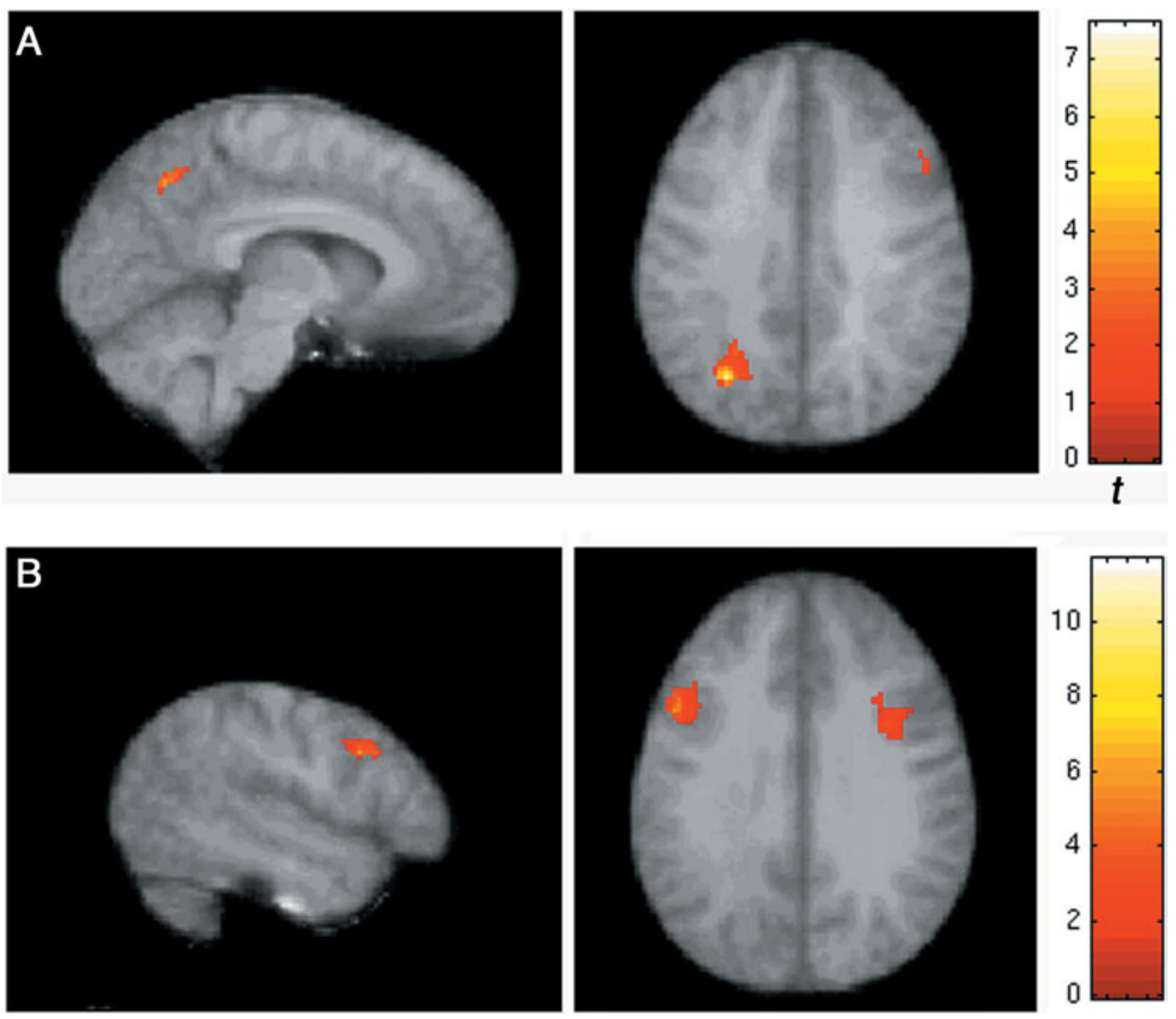

Fig. 3. Statistical Parametric Mapping (SPM) within-groups results, inverted face task. A: (top) shows ASD subjects with unique activation in the precuneus and reduced activation in right prefrontal cortex. B: (bottom) shows TD children with prominent activation in prefrontal cortex (left) for inverted faces.

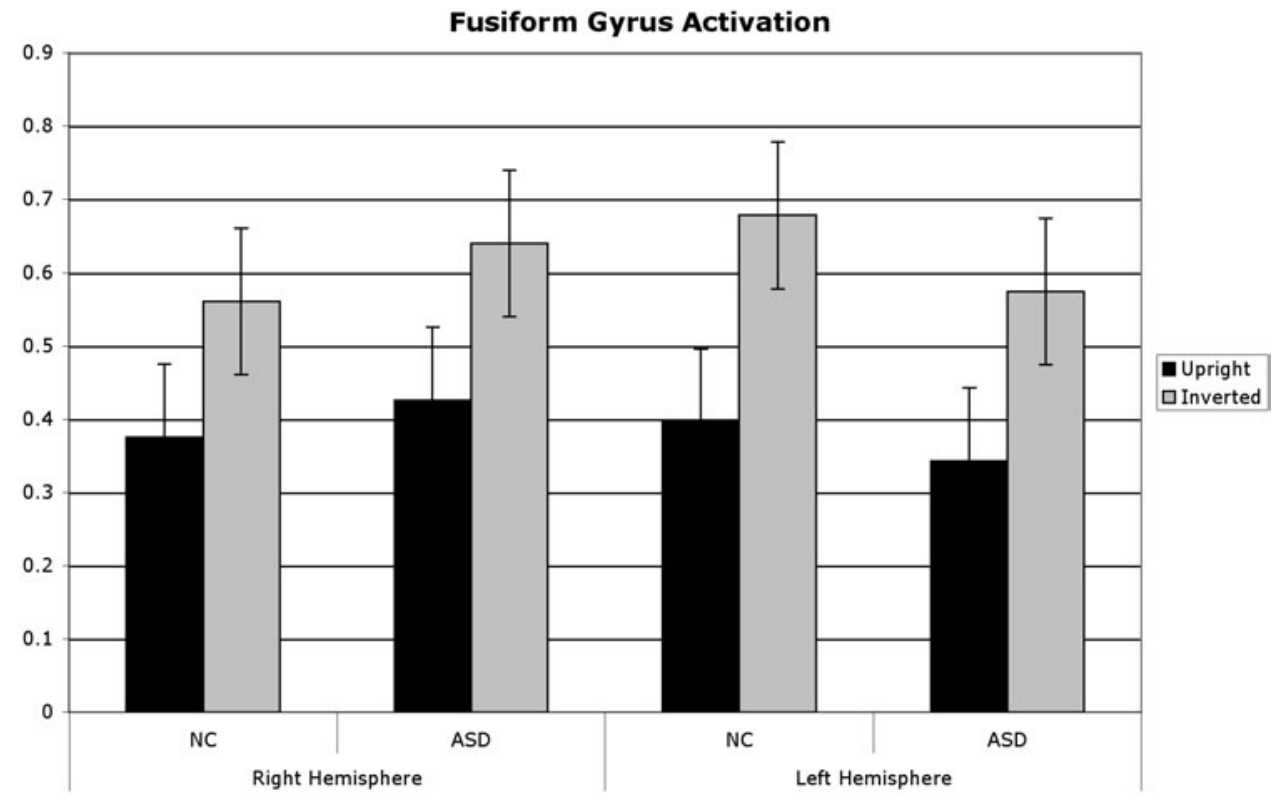

Fig. 4. Fusiform gyrus ROI values. There was a main effect of task with more fMRI activation for inverted compared with upright faces, but no differences between control and ASD children. 


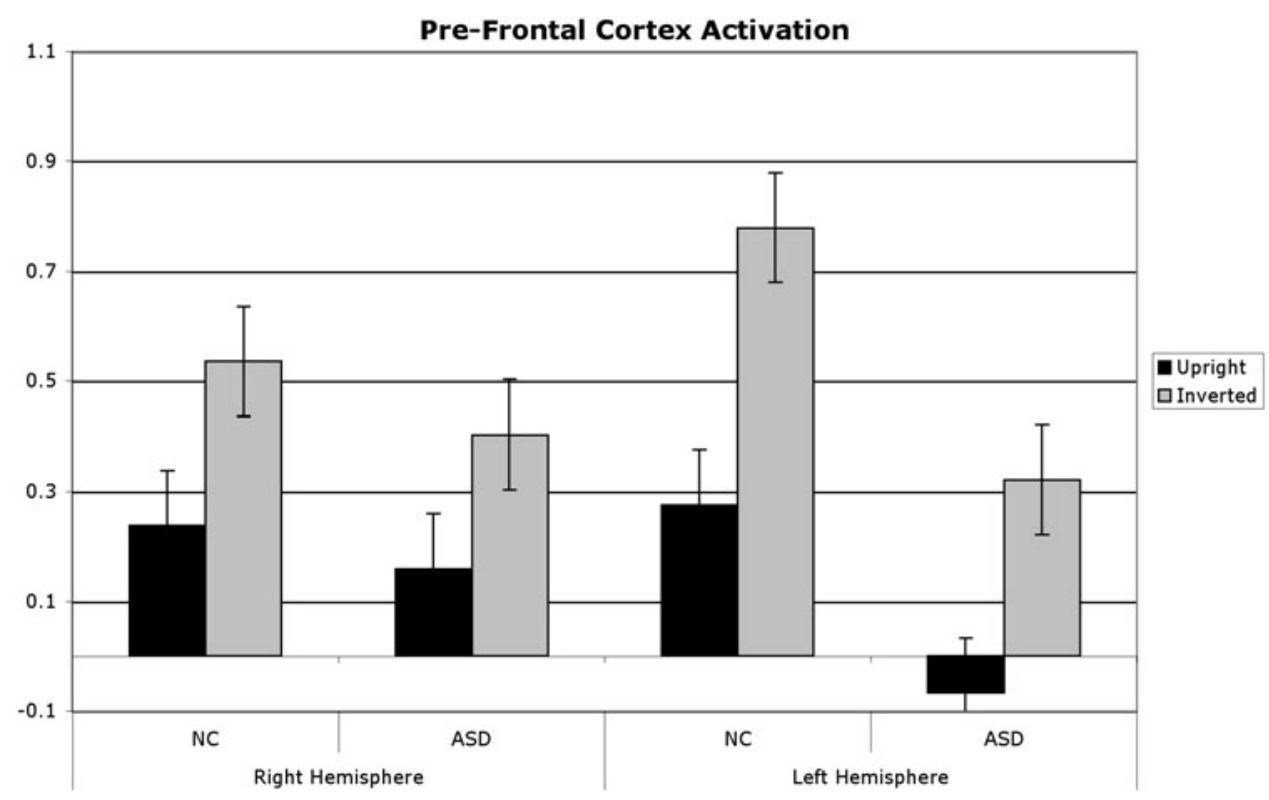

Fig. 5. Prefrontal cortex ROIs demonstrate a main effect for task, with more activation for inverted compared with upright faces and a group $\times$ hemisphere interaction, with greater PFC activation for control compared with ASD children in the left hemisphere; right hemisphere differences showed a nonsignificant trend in the same direction.

tested the effects of same variables on right fusiform activation, because differences among autistic individuals in this region are frequently reported.

Across all subjects (ASD and TD), there was a marginally significant correlation between age and activation in the right fusiform ROI $(r=.39$, one-tailed $p<.05$, uncorrected), suggesting that activation magnitude in the right fusiform during upright face processing tended to increase with age (there were no age differences between ASD and TD groups). In the left IFG, there was no relationship between age and activation magnitude $(r=.26 ; p>.2)$. Within the ASD group, there were no correlations between scores on the ADOS and activation in either the LPFC ( $r=$ .12 ) or the fusiform gyrus ( $r=.07)$; however, we note that the range of severity for ASD children on all measures was very narrow.

The magnitude of the inversion effect, based on the difference in reaction time between upright and inverted face performance, showed a small but nonsignificant correlation with activation during upright face processing in the left PFC ( $r=.26 ; p>.1$ uncorrected). An apparently significant (uncorrected) correlation with the right fusiform ( $r=$ $.53 ; p>.02$ was driven by one ASD subject performing at chance, and was much reduced when that subject was removed $(r=.26 ; p>.2)$. Correlation of simple reaction time during upright face processing with activity in the left PFC was similarly nonsignificant $(r=-.21 ; p>.2)$, suggesting that differences in behavioral performance could not explain the significant group difference in activation in the left PFC. In the right fusiform, the correlation between reaction time and activation was -.44 , marginally significant $(p=.05)$ without correction for multiple comparisons.

\section{DISCUSSION}

Consistent with prior behavioral studies of the inversion effect in ASD, our typically developing children showed a larger performance decrement for inverted compared with upright faces than did the children with autism. This replicates prior findings in the literature showing a reduced or absent inversion effect in ASD while confirming a significant inversion effect in typically developing children. However, this effect was due not to superior performance in ASD for inverted faces, but rather to relatively impaired performance on upright faces. It is important to note that the performance results are based on reaction time, not accuracy data, as the latter showed strong ceiling effects in this task. FMRI results showed that group differences were found in the frontal cortex for both upright and inverted tasks. Moreover, we did not detect differences between ASD and TD children in the fusiform gyrus region for either condition. Rather, the significant differences in brain activity between groups were found in inferior and middle frontal brain regions as well as in the amygdala. These data suggest that key differences in face processing in children with autism are due to differences in brain regions involving fronto-limbic systems. Our data are inconsistent with the notion that the inversion effect can be explained by altered activity in the FFA, because FFA activity was the same between ASD and TD groups. Instead, the brain areas that differentiated ASD from TD children during both of the face processing tasks were found primarily in several brain regions typically associated with the so-called "social brain: (Hadjikhani et al., 2007; Frith, 2007). In frontal cortex, activation was extensive for typically developing children 
and was more active than in ASD children for all conditions. The most unique areas of activation differences were found in the left hemisphere, in the dorsal portion of the inferior frontal gyrus. Aside from its role on language, this region has been reported in studies of "mirror neuron" activity in normal adults (Iacoboni, 2005), and is less active during imitation and observation of faces in children with autism compared to TD children (Dapretto et al., 2006). The "mirror neuron" areas, including frontal and limbic brain regions, play a specific role in processing meaningful, goal directed behaviors that are observed in others by effectively mirroring those behaviors internally, and appear to be related to social cognition (Iacoboni \& Dapretto, 2006). Hadjikhani et al. (2007) also found reduced activity in the IFG during face processing in autism though in the right hemisphere, while Dapretto et al. (2006) found bilateral reductions in this region among ASD children. Structural abnormalities of the IFG have also been reported in autism (Hadjikhani et al., 2006). Together these data suggest that IFG dysfunction is a consistent finding in autism spectrum disorders.

Another region associated with the "social brain" network is the amygdala. While prior studies have noted amygdala differences between ASD and TD children when observing faces bearing emotional expressions (e.g., Pelphrey et al., 2007; Piggot et al., 2004; Wang et al., 2004), the stimuli used in the present study were of neutral expression suggesting that face stimuli may automatically invoke neural activity in this region among TD children. The IFG is thought to project to the amygdala via the insula in a pathway that may add an emotional valence to processing the intentions of others (Carr et al., 2003; Dapretto et al., 2006). Abnormal fronto-limbic development in children with autism may contribute to their deficits in social cognition.

While the interpretation of the inversion effect in autism remains controversial, one argument is that ASD children do not visually process faces as unique entities, instead perceiving them similarly to other objects (Hobson et al., 1988), and thus are less hampered by inversion. Alternatively, ASD children may typically engage more in component rather than configural processing, a strategy that could be applied across stimulus types but may be better suited for inverted faces (Ashwin et al., 2006). The hypothesis that the fusiform face area typically processes information configurally has been challenged in recent fMRI studies. Yovel and Kanwisher (2004) argued that in normal adults, face processing takes place uniquely in the FFA regardless of whether subjects apply a configural or parts-based task, and regardless of whether the faces are upright or inverted (Yovel \& Kanwisher, 2005), though activation was somewhat greater for upright faces. Other studies have found no difference between upright and inverted faces in the FFA (Aguirre et al., 1999; Haxby et al., 1999). Prior studies have shown mixed results on whether ASD children show abnormal responses in the FFA. While many earlier studies found reduced FFA activity in ASD (Pierce et al., 2001; Schultz et al., 2000) others, particularly those in which the faces were familiar to the subjects (Pierce et al., 2004) or when the tasks required subjects to maintain fixation on the faces showed no FFA differences (Pelphrey et al., 2005; Piggot et al., 2004; Wang et al., 2004). In the present study, the task demands to match the faces in the inverted condition required subjects to remain focused on the face stimuli; avoiding the face would be expected to impair performance significantly. Although ASD subjects performed slightly worse than control subjects for upright faces, their overall accuracy of $93 \%$ indicates they performed the task well and thus must have processed the faces. Therefore, we cannot easily attribute the differential behavioral inversion effect to differences in visual aspects of face processing in the present study.

We found greater activation in all regions of interest for the inverted task. This finding may relate to the increased difficulty of the inverted task generally. Prior studies of task difficulty show increases in fMRI activation, more specifically in frontal cortex and sensory processing regions, as task demands increase (e.g., Burggren et al., 2002; Kroger et al., 2002). However, post hoc analyses correlating task performance with fMRI responses found no evidence that performance differences between subjects could account for the selective group differences in PFC activation in our study.

Of interest, one brain area in which ASD subjects showed increased activity compared with controls was the precuneus. Prior imaging studies of ASD using a range of paradigms have also implicated this region (Wang et al., 2004), which is thought to be a primary part of the "resting" brain network (Raichle et al., 2001). The significance of this network is not well understood; it appears to be consistently reduced in activity when subjects engage in any kind of effortful task, and increases during rest (Raichle et al., 2001; Raichle \& Snyder, 2007). Increased activation in this region could reflect a failure of children with autism to move away from a resting or internally directed state. Further studies focusing on this region, including resting-state studies, may help to elucidate the role of this region in autism.

There are several limitations of this study. A ubiquitous potential confound in face processing studies is that ASD subjects may tend to avert their gaze or spend less time looking at the face stimuli (Klin et al., 2002); this may result in decrease activity in the face area (Dalton et al., 2005). While we were unable to record eye movements in the scanner, the nature of the task we used demanded prolonged exposure and/or attention to the faces; thus, the tendency for ASD subjects to avoid looking at the faces would be lessened, as they could not achieve adequate performance if they averted their gaze. If they had averted gaze, we would expect less activation in the face areas, whereas we found equivalent activation magnitudes in this region.

Another potential limitation is the number of subjects; while 12 subjects per group is considered sufficient for standard comparisons, the heterogeneity of autism makes it possible that we failed to observe subgroups with unique patterns 
of responses. One study found different inversion effects for those with more general face processing deficits but typical effects for those without severe face processing problems (Teunisse \& de Gelder, 2003). It is possible that a heterogenous sample may mask deficits in visual information processing seen in some studies of autism.

Finally, a strong ceiling effect in accuracy for the TD group precluded our performing analysis on accuracy data. The majority of face inversion studies use accuracy rather than reaction time to determine inversion effects (e.g., Freire et al., 2000; Yin, 1969). However, similar inversion effects for reaction time and accuracy have been reported in prosopagnosic patients (Farah et al., 1995b) as well as in children with autism (Ashwin et al., 2006), suggesting that the results from RT and accuracy measures may be comparable.

In summary, our study corroborates prior research showing a reduced inversion effect during face processing in autism; however, our data suggest this is due to impaired face processing generally, not enhanced inverted face processing as has been suggested previously. Neuroanatomically, we find no evidence that activation differences in the fusiform gyrus underlie this effect. Rather, our findings suggest that face processing differences that underlie the differential inversion effect in autism spectrum disorders are primarily represented in frontal cortex and in the amygdala, and thus appear to reflect differences in processing the meaning and significance of faces. This study adds to a growing body of evidence from structural (Hadjikhani et al., 2006) and functional (e.g., Dapretto et al., 2006; Hadjikhani et al., 2007) imaging implicating top-down mechanisms for abnormal face processing in autism.

\section{ACKNOWLEDGMENTS}

This study was funded in part by NICHD grants 5U19HD035470:10 and 1P50-HD055784:1 to Marian Sigman, Susan Bookheimer, and Mirella Dapretto. The authors have no other financial relationships that could constitute a conflict of interest.

\section{REFERENCES}

Aguirre, G.K., Singh, R., \& D'Esposito, M. (1999). Stimulus inversion and the responses of face and object-sensitive cortical areas. Neuroreport, 10, 189-194.

Ashwin, C., Wheelwright, S., \& Baron-Cohen, S. (2006). Finding a face in the crowd: Testing the anger superiority effect in Asperger Syndrome. Brain and Cognition, 61, 78-95.

Barton, J.J., Hefter, R.L., Cherkasova, M.V., \& Manoach, D.S. (2007). Investigations of face expertise in the social developmental disorders. Neurology, 69, 860-870.

Barton, J., Keenan, J., \& Bass, T. (2001). Discrimination of spatial relations and features in faces: Effects of inversion and viewing duration. British Journal of Psychology, 92, 527-549.

Behrmann, M., Avidan, G., Leonard, G.L., Kimchi, R., Luna, B., Humphreys, K., \& Minshew, N. (2006). Configural processing in autism and its relationship to face processing. Neuropsychologia, 44, 110-129.
Berument, S.K., Rutter, M., Lord, C., Pickles, A., \& Bailey, A. (1999). Autism screening questionnaire: Diagnostic validity. British Journal of Psychiatry, 175, 444-451.

Burggren, A.C., Small, G.W., Sabb, F.W., \& Bookheimer, S.Y. (2002). Specificity of brain activation patterns in people at genetic risk for Alzheimer's disease. American Journal of Geriatric Psychiatry, 10, 44-51.

Campbell, R., Lawrence, K., Mandy, W., Mitra, C., Jeyakuma, L., \& Skuse, D. (2006). Meanings in motion and faces: Developmental associations between the processing of intention from geometrical animations and gaze detection accuracy. Development and Psychopathology, 18, 99-118.

Carr, L., Iacoboni, M., Dubeau, M.C., Mazziotta, J.J., \& Lenzi, G.L. (2003). Neural mechanisms of empathy in humans: A relay from neural systems for imitation to limbic areas. Proceedings of the National Academy of Science of the United States of America, 100, 5497-5502.

Chawarska, K., Klin, A., \& Volkmar, F. (2003). Automatic attention cueing through eye movement in 2-year-old children with autism. Child Development, 74, 1108-1122.

Dalton, K.M., Nacewicz, B.M., Johnstone, T., Schaefer, H.S., Gernsbacher, M.A., Goldsmith, H.H., Alexander, A.L., \& Davidson, R.J. (2005). Gaze fixation and the neural circuitry of face processing in autism. Nature Neuroscience, 8 , 519-526.

Dapretto, M., Davies, M.S., Pfeifer, J.H., Scott, A.A., Sigman, M., Bookheimer, S.Y., \& Iacoboni, M. (2006). Understanding emotions in others: Mirror neuron dysfunction in children with autism spectrum disorders. Nature Neuroscience, 9, 28-30.

Dawson, G., Webb, S.J., Wijsman, E., Schellenberg, G., Estes, A., Munson, J., \& Faja, S. (2005). Neurocognitive and electrophysiological evidence of altered face processing in parents of children with autism: Implications for a model of abnormal development of social brain circuitry in autism. Development and Psychopathology, 17, 679-697.

Dunn, L.M. \& Dunn, L.M. (1997). Peabody Picture Vocabulary Test: III. Circle Pines, MN: American Guidance Service.

Ekman, P. \& Friesen, W. (1976). Measuring facial movement. Environmental Psychology and Nonverbal Behavior, 1, 56-75.

Farah, M., McMullen, P., \& Meyer, M. (1991). Can recognition of living things be selectively impaired? Neuropsychologia, 29, 185-193.

Farah, M.J., Tanaka, J.W., \& Drain, H.M. (1995a). What causes the face inversion effect? Journal of Experimental Psychology. Human Perception and Performance, 21, 628-634.

Farah, M.J., Wilson, K.D., Drain, H.M., \& Tanaka, J.R. (1995b). The inverted face inversion effect in prosopagnosia: Evidence for mandatory, face-specific perceptual mechanisms. Vision Research, 35, 2089-2093.

Freire, A., Lee, K., \& Symons, L.A. (2000). The face-inversion effect as a deficit in the encoding of configural information: Direct evidence. Perception, 29, 159-170.

Frith, U. (2007). The social brain? Philosophical Transactions of the Royal Society of London. Series B, Biological Sciences, 362, 671-678.

Hadjikhani, N., Joseph, R.M., Snyder, J., Chabris, C.F., Clark, J., Steele, S., McGrath, L., Vangel, M., Aharon, I., Feczko, E., Harris, G.J., \& Tager-Flusberg, H. (2004). Activation of the fusiform gyrus when individuals with autism spectrum disorder view faces. Neuroimage, 22, 1141-1150.

Hadjikhani, N., Joseph, R.M., Snyder, J., \& Tager-Flusberg, H. (2006). Anatomical differences in the mirror neuron system 
and social cognition network in autism. Cerebral Cortex, 16, 1276-1282.

Hadjikhani, N., Joseph, R.M., Snyder, J., \& Tager-Flusberg, H. (2007). Abnormal activation of the social brain during face perception in autism. Human Brain Mapping, 28, 441-449.

Happe, F. \& Frith, U. (2006). The weak coherence account: Detailfocused cognitive style in autism spectrum disorders. Journal of Autism and Developmental Disorders, 36, 5-25.

Haxby, J.V., Ungerleider, L.G., Clark, V.P., Schouten, J.L., Hoffman, E.A., \& Martin, A. (1999). The effect of face inversion on activity in human neural systems for face and object perception. Neuron, 22, 189-199.

Hobson, R.P., Ouston, J., \& Lee, A. (1988). What's in a face? The case of autism. British Journal of Psychology, 79(Pt 4), 441-453.

Iacoboni, M. (2005). Neural mechanisms of imitation. Current Opinion in Neurobiology, 15, 632-637.

Iacoboni, M. \& Dapretto, M. (2006). The mirror neuron system and the consequences of its dysfunction. Nature Reviews. Neuroscience, 7, 942-951.

Klin, A., Jones, W., Schultz, R., Volkmar, F., \& Cohen, D. (2002). Visual fixation patterns during viewing of naturalistic social situations as predictors of social competence in individuals with autism. Archives of General Psychiatry, 59, 809-816.

Kroger, J.K., Sabb, F.W., Fales, C., Bookheimer, S.Y., Cohen, M.S., \& Holyoak, K.J. (2002). Recruitment of anterior dorsolateral prefrontal cortex in human reasoning: A parametric study of relational complexity. Cerebral Cortex, 12, 477-485.

Lord, C., Risi, S., Lambrecht, L., Cook, E.H., Jr., Leventhal, B.L., DiLavore, P.C., Pickles, A., \& Rutter, M. (2000). The autism diagnostic observation schedule-generic: A standard measure of social and communication deficits associated with the spectrum of autism. Journal of Autism and Developmental Disorders, 30, 205-223.

Lord, C., Rutter, M., \& Le Couteur, A. (1994). Autism Diagnostic Interview-Revised: A revised version of a diagnostic interview for caregivers of individuals with possible pervasive developmental disorders. Journal of Autism and Developmental Disorders, 24, 659-685.

Mottron, L., Belleville, S., \& Menard, E. (1999). Local bias in autistic subjects as evidenced by graphic tasks: Perceptual hierarchization or working memory deficit? Journal of Child Psychology and Psychiatry, and Allied Disciplines, 40, 743-755.

Pellicano, E., Jeffery, L., Burr, D., \& Rhodes, G. (2007). Abnormal adaptive face-coding mechanisms in children with autism spectrum disorder. Current Biology, 17, 1508-1512.

Pelphrey, K.A., Morris, J.P., \& McCarthy, G. (2005). Neural basis of eye gaze processing deficits in autism. Brain, 128(Pt 5), 1038-1048.

Pelphrey, K.A., Morris, J.P., McCarthy, G., \& Labar, K.S. (2007). Perception of dynamic changes in facial affect and identity in autism. Social Cognitive and Affective Neuroscience, 2, 140-149.
Pierce, K., Haist, F., Sedaghat, F., \& Courchesne, E. (2004). The brain response to personally familiar faces in autism: Findings of fusiform activity and beyond. Brain, 127(Pt 12), 2703-2716.

Pierce, K., Muller, R.A., Ambrose, J., Allen, G., \& Courchesne, E. (2001). Face processing occurs outside the fusiform 'face area' in autism: Evidence from functional MRI. Brain, 124(Pt 10), 2059-2073.

Piggot, J., Kwon, H., Mobbs, D., Blasey, C., Lotspeich, L., Menon, V., Bookheimer, S., \& Reiss, A.L. (2004). Emotional attribution in high-functioning individuals with autistic spectrum disorder: A functional imaging study. Journal of the American Academy of Child and Adolescent Psychiatry, 43, 473-480.

Raichle, M.E., MacLeod, A.M., Snyder, A.Z., Powers, W.J., Gusnard, D.A., \& Shulman, G.L. (2001). A default mode of brain function. Proceedings of the National Academy of Sciences of the United States of America, 98, 676-682.

Raichle, M.E. \& Snyder, A.Z. (2007). A default mode of brain function: A brief history of an evolving idea. Neuroimage, 37, 1083-1090; discussion 1097-1099.

Rose, F.E., Lincoln, A.J., Lai, Z., Ene, M., Searcy, Y.M., \& Bellugi, U. (2007). Orientation and affective expression effects on face recognition in Williams syndrome and autism. Journal of Autism and Developmental Disorders, 37, 513-522.

Schultz, R.T., Gauthier, I., Klin, A., Fulbright, R.K., Anderson, A.W., Volkmar, F., Skudlarski, P., Lacadie, C., Cohen, D.J., \& Gore, J.C. (2000). Abnormal ventral temporal cortical activity during face discrimination among individuals with autism and Asperger syndrome. Archives of General Psychiatry, 57, 331-340.

Talairach, J. \& Tournoux, P. (1988). Co-Planar Stereotaxic Atlas of the Human Brain. New York: Thieme Medical Publishers.

Teunisse, J.P. \& de Gelder, B. (2003). Face processing in adolescents with autistic disorder: The inversion and composite effects. Brain and Cognition, 52, 285-294.

Wang, A.T., Dapretto, M., Hariri, A.R., Sigman, M., \& Bookheimer, S.Y. (2004). Neural correlates of facial affect processing in children and adolescents with autism spectrum disorder. Journal of the American Academy of Child and Adolescent Psychiatry, 43, 481-490.

Wechsler, D. (1991). Wechsler Intelligence Scale for ChildrenThird Edition. San Antonio, TX: The Psychological Corporation.

Woods, R., Grafton, S., Holmes, C., Cherry, S., \& Mazziotta, J. (1998). Automated image registration: I. General methods and intrasubject, intramodality validation. Journal of Computer Assisted Tomography, 22, 141-154.

Yin, R.K. (1969). Looking at upside-down faces. Journal of Experimental Psychology, 81, 141-145.

Yovel, G. \& Kanwisher, N. (2004). Face perception: Domain specific, not process specific. Neuron, 44, 889-898.

Yovel, G. \& Kanwisher, N. (2005). The neural basis of the behavioral face-inversion effect. Current Biology, 15, 2256-2262. 